\title{
Portal Hypertension in Non-alcoholic Fatty Liver Disease in the Era of Non-invasive Assessment
}

\author{
Authors: \\ *Cosmas Rinaldi Adithya Lesmana,,2 Maria Satya Paramitha," Irsan \\ Hasan, ${ }^{1}$ Andri Sanityoso Sulaiman, ${ }^{1}$ Rino Alvani Gani \\ 1. Department of Internal Medicine, Hepatobiliary Division, Dr. Cipto Mangunkusumo \\ National General Hospital, Universitas Indonesia, Jakarta, Indonesia \\ 2. Digestive Disease \& GI Oncology Center, Medistra Hospital, Jakarta, Indonesia \\ *Correspondence to medicaldr2001id@yahoo.com
}

Disclosure:

The authors have declared no conflicts of interest.

Received:

13.02.21

Accepted:

21.07.21

Keywords:

Non-alcoholic fatty liver disease (NAFLD), portal hypertension, portal pressure measurement.

Citation:

EMJ. 2021;6[3]:80-93

\section{Abstract}

Non-alcoholic fatty liver disease (NAFLD) is one of the emerging global health problems due to an increase of burden worldwide. It has been known that NAFLD is strongly associated with metabolic syndrome. The progression of NAFLD is a complex and multifactorial mechanism. Portal hypertension is still the main key in liver disease progression management. In NAFLD, portal hypertension might occur in the non-cirrhotic condition. Hepatic vein pressure gradient measurement has been considered as the gold standard for portal pressure assessment; however, due to its invasiveness and the need for a high-expertise centre, it is considered a non-practical measurement tool in clinical practice. Many other non-invasive parameters have been developed to replace the invasive measurement; however, there are still some limitations with regard to the technical issue, patient's condition, and its accuracy in the different stages of the disease. Therefore, the authors review portal hypertension related to the clinical course of NAFLD, and the development of portal pressure evaluation in patients with NAFLD.

\section{INTRODUCTION}

Non-alcoholic fatty liver disease (NAFLD) is considered as one of the fastest emerging global health priorities due to the continuous increase of burden worldwide. ${ }^{1,2}$ The progression of NAFLD itself is a complex and multifactorial mechanism. A progressive liver disease in NAFLD is marked by hepatocyte injury in the form of ballooning, inflammation, and fibrosis, which is also known as non-alcoholic steatohepatitis (NASH). ${ }^{3}$

In NAFLD progression, one of the most common complications is portal hypertension due to extensive fibrosis with parenchymal and vascular remodelling in cirrhotic liver. Over the last two decades, the use of hepatic venous pressure gradient (HVPG) has become the most utilised measurement for portal pressure. This method, however, has been limited by the fact that it is an invasive technique and can only be conducted in highly specialised medical centres. Moreover, increasing demand for non-invasive methods is also contributed by the problem of early progression from intrahepatic vascular resistance to liver disease, even in the absence of cirrhosis. ${ }^{4,5}$ Therefore, development of safe and reliable non-invasive methods for measurement and 
monitoring of portal pressure is still important for prevention and early management in NAFLDrelated portal hypertension.

\section{AIM OF THE STUDY}

This review will discuss the mechanisms behind NAFLD-related portal hypertension in the presence or absence of cirrhosis. Recent findings about non-invasive diagnostic modalities for portal hypertension, especially those related to the potential use in non-cirrhotic portal hypertension, will also be reviewed.

\section{CLINICALLY SIGNIFICANT PORTAL HYPERTENSION IN NON-ALCOHOLIC FATTY LIVER DISEASE}

Clinically significant increased portal pressure or clinically significant portal hypertension (CSPH) is defined as an increase of portohepatic gradient of at least $10 \mathrm{mmHg}$. Determination of $\mathrm{CSPH}$ is critical to evaluate possible complications, such as oesophageal varices or ascites, because different levels of risk and prognostic significance are represented by the degree of portal hypertension. ${ }^{6,7}$ Portal pressure higher than $20 \mathrm{mmHg}$ is also correlated with difficult variceal bleeding management. ${ }^{8}$

In general, portal hypertension is caused by increased resistance towards portal blood flow. Increase in hepatic vascular resistance is caused by either structural component or dynamic component in the form of increased hepatic vascular tone. Increase in portal-collateral blood flow is caused by splanchnic arteriolar vasodilation and neo-angiogenesis as a response towards increased production of splanchnic vasodilators. ${ }^{9}$ In NAFLD, structural and functional changes, such as enlarged hepatocytes and ballooning injury, may affect the homeostasis of sinusoids since the early stage of the disease through endothelial dysfunction, disrupted sinusoidal microanatomy, and cross-talks among hepatocytes. As a result, intravascular hepatic resistance will be increased, leading to progression of NAFLD and development of portal hypertension. To sum up, development of portal hypertension in NAFLD can occur through early sinusoidal compression and microcirculatory disruption without the presence of extensive fibrosis or tissue remodelling in cirrhosis. 8,10
As mentioned above, another important problem in NAFLD-related portal hypertension is the concept of portal hypertension without the presence of fibrosis or cirrhosis. A prospective study of 292 subjects with NAFLD showed that $17 \%$ of the subjects who did not have cirrhosis were found to have portal hypertension (HVPG $>5 \mathrm{mmHg}$ ), in which $0.5 \%$ of the subjects had CSPH. ${ }^{11}$ Another observational study of 354 subjects with NAFLD also demonstrated similar findings, showing that 12 out of 100 subjects with portal hypertension (only based on clinical manifestation) did not exhibit any advanced fibrosis or cirrhosis. The authors also pointed out that the only significant difference between subjects with and without portal hypertension was the severity of steatosis. ${ }^{12}$ In contrast, a retrospective study of 261 subjects showed no significant association between histological steatosis and HVPG measurement. However, this study also showed that the presence of diabetes mellitus, one of the most predominant risk factors of NAFLD, was significantly associated with the presence of $\mathrm{CSPH} .{ }^{13}$ Consequently, development of non-invasive modalities that can be a prognostic indicator related to the presence of portal hypertension in patients without advanced fibrosis or cirrhosis still becomes a necessity.

\section{ASSESSMENT OF PORTAL HYPERTENSION IN NON-ALCOHOLIC FATTY LIVER DISEASE WITHOUT CIRRHOSIS}

The studies in patients with NAFLD and without cirrhosis, especially the ones utilising large-scale -omics technologies and integrative systems biology, are still considered as very scarce. An observational study by Da et al. ${ }^{14}$ of patients with non-cirrhotic portal hypertension indicated that no significant difference was found among HVPG results of patients with portal hypertension caused by nodular regenerative hyperplasia. Unlike in cirrhosis, nodular regenerative hyperplasia often results in perisinusoidal or presinusoidal portal hypertension, which often show normal or mildly increased HVPG. ${ }^{14}$ Other possible reasons behind the drawback of these studies may include sporadic utilisation of portal pressure measurement, especially considering its invasive nature, such as HVPG measurement, in patients with less-advanced early disease. Consequently, higher number of non-invasive 
options are still necessary to overcome the lack of portal pressure measurement methods in patients with early stages of advanced liver disease..$^{10,14}$

Magnetic resonance (MR)-based methods have demonstrated the ability to discern between portal hypertension with and without cirrhosis. In a retrospective evaluation of 41 subjects with non-cirrhotic portal hypertension, magnetic resonance elastography (MRE) also indicated a promising result, showing that increased liver stiffness measurement, as well as increased ratio of splenic stiffness measurement and liver stiffness measurement, can distinguish non-cirrhotic portal hypertension from cirrhotic portal hypertension. The study also showed that liver stiffness measurement was markedly lower in portal hypertension without cirrhosis, while the ratio between spleen stiffness measurement and liver stiffness measurement was markedly higher in portal hypertension without cirrhosis. ${ }^{15}$ In addition, MR-based methods have been well-correlated with a wide range of HVPG measurements (3-16 mmHg). A study by Gharib et al. ${ }^{16}$ demonstrated an independent significant correlation ( $p=0.015)$ between HVPG and MRE of the liver, with a median HVPG of $6 \mathrm{mmHg}$ from 23 subjects. Liver stiffness measurement by MRE also showed significant correlation with histologic fibrosis score $(p=0.004) .{ }^{16}$

The emerging use of non-invasive serum biomarkers, especially metabolite profiling, which incorporates genetic and environmental inputs, has been summarised in previous studies. This is particularly in line with the fact that both genetic and environmental factors play a very important role in NAFLD progression. In addition, markers of metabolic status and microbiome changes can also act as early predictors of portal hypertension in NAFLD since the pathophysiology of NAFLD also involves gut microbiota changes and metabolic syndrome. ${ }^{10}$ Generally, the aim of metabolomics or metabolic profiling is to measure endogenous small metabolites quantitatively. Since the measured small molecular metabolites contain substrates and by-products, such as carbohydrates, fatty acids, and amino acids, the results may represent the metabolic responses in a disease or potential intermediate phenotypes. ${ }^{17}$ On the other hand, soluble CD163 scavenger receptor and enzyme haem oxygenase-1 have been shown as Kupffer cell-specific markers, related to the microbiota disturbance in NAFLD. ${ }^{10}$ Activation of Kupffer cells and bacterial translocation occur due to increased intestinal permeability. Activated Kupffer cells will eventually lead to increased production of inflammatory mediators and activation of hepatic stellate cells, leading to liver fibrosis. A study by Grønbaek et al. ${ }^{18}$ also highlighted a significant correlation between circulating soluble CD163 and HVPG, indicating its role as an independent predictor for HVPG. ${ }^{18}$ Other proteins associated with intrahepatic endothelial dysfunction and remodelling of extracellular matrix include von Willebrand factor antigen, the formation marker procollagen Type $\mathrm{V}$, and osteopontin. ${ }^{19}$ Overall, these novel biomarkers, especially when they are being measured simultaneously and/or serially, can be potentially advantageous in predicting disease progression, even in early stage diagnosis. ${ }^{10}$

\section{ASSESSMENT OF PORTAL HYPERTENSION IN NON-ALCOHOLIC FATTY LIVER DISEASE WITH CIRRHOSIS}

Portal pressure gradient (PPG) is known as the pressure gradient between the portal vein and inferior vena cava. Measurement of PPG represents measurement of liver portal perfusion pressure. The normal value is up to $5 \mathrm{mmHg}{ }^{9,10}$

\section{Hepatic Venous Pressure Gradient}

Currently, HVPG measurement is considered as the gold standard technique to assess portal hypertension in liver cirrhosis. HVPG measurement is defined as the measurement of difference between wedged hepatic venous pressure and normal free hepatic venous pressure. ${ }^{4,9}$ HVPG measurement is considered a safe technique due to its low rate of complications $(<1 \%$ of the cases, mainly caused by transient cardiac arrhythmias and local injury on the site of puncture). This technique also does not have many relative contraindications, such as abnormal coagulation parameters and history of allergic reactions towards iodinated contrast. ${ }^{20}$ In addition, HVPG measurement has also been considered as one of the best prognostic indicators in liver cirrhosis (Table 1). ${ }^{6}$ 
Table 1: Prognostic stratification of liver cirrhosis by hepatic venous pressure gradient measurement. ${ }^{6-9}$

\begin{tabular}{|l|l|l|}
\hline Clinical Settings & HVPG $(\mathbf{m m H g})$ & Increased risk of threshold \\
\hline Compensated cirrhosis & 10 & $\begin{array}{l}\text { Gastroesophageal varices, first episode of clinical } \\
\text { decompensation in patients without varices, development of } \\
\text { HCC, decompensation post-surgery for HCC }\end{array}$ \\
\cline { 2 - 4 } & 12 & Variceal bleeding \\
\cline { 2 - 4 } & 16 & First episode of decompensation in patients with varices \\
\hline Decompensated cirrhosis & 16 & Variceal rebleeding \\
\cline { 2 - 4 } & 20 & Failure of controlling active variceal bleeding \\
\cline { 2 - 3 } & 22 & Mortality in alcoholic cirrhosis and acute alcoholic hepatitis \\
\cline { 2 - 4 } & 30 & Spontaneous bacterial peritonitis \\
\hline Acute variceal bleeding & 20 & Treatment failure and increased mortality \\
\hline
\end{tabular}

HCC: hepatocellular carcinoma; HVPG: hepatic vein pressure gradient.

\section{Upper Gastrointestinal Endoscopy}

Another gold standard for evaluating oesophageal and gastric varices with the bleeding risk is an upper gastrointestinal endoscopy or oesophagogastroduodenoscopy (OGD). Oesophageal varices have been estimated to be present in $30-40 \%$ of compensated cirrhosis and up to $60 \%$ of decompensated cirrhosis.9,21 According to Baveno VI criteria, high-risk varices are considered to be unlikely in patients with liver stiffness measurement $<20 \mathrm{kPa}$ from the result of transient elastography (TE) and normal platelet count. 22,23 Based on these criteria, newly diagnosed patients with liver cirrhosis must undergo an OGD examination to exclude the presence of gastroesophageal varices. ${ }^{20,24}$

\section{Video Capsule}

Video capsule is one of the applications of endoscopy-guided technique to overcome the invasive characteristic of an OGD. A multi-centre study conducted by Laurain et al. ${ }^{25}$ showed that the sensitivity, specificity, positive predictive value (PPV), and negative predictive value (NPV) of video capsule were 65\%, 83\%, $65 \%$, and $83 \%$, respectively. The diagnostic values of video capsule were lower when it was used to differentiate between small and large oesophageal varices (sensitivity: 64\%; specificity: 93\%; PPV: 88\%; NPV: 78\%; and overall accuracy: 81\%). Taken together, it was concluded that the diagnostic values of video capsule were inadequate to replace OGD in patients with cirrhosis with clinical suspicion of portal hypertension. ${ }^{26}$

\section{Non-invasive Modalities in the Diagnosis of Non-alcoholic Fatty Liver Disease-Related Portal Hypertension with Cirrhosis}

\section{Ultrasonography}

The pathognomonic signs in portal hypertension are the presence of flow in paraumbilical vein and splenorenal collaterals, as well as reversed flow of the portal vein. Another grey-scale sign of portal hypertension is dilation of portal, mesenteric, and splenic veins. There are two ultrasound-based modalities commonly used for detecting portal hypertension: Doppler ultrasound and contrast-enhanced ultrasound (CEUS). ${ }^{20,27}$ Different sensitivities and specificities from different signs of portal hypertension from ultrasound-based modalities are summarised in Table $2 .^{5}$ 
Table 2: Summary of sensitivity and specificity values from each sign of portal hypertension obtained from ultrasound-based modalities. $^{5}$

\begin{tabular}{|c|c|c|c|}
\hline Signs of portal hypertension & References & Sensitivity (\%) & Specificity (\%) \\
\hline Dilation of portal vein (>13 mm) & Bolondi et al.,34 1982 & $<50.0$ & $90.0-100.0$ \\
\hline Reduction of portal vein blood flow velocity & $\begin{array}{l}\text { Zironi et al.,35 } 1992 \\
\text { Haag et al., }{ }^{36} 1999\end{array}$ & 88.0 & 96.0 \\
\hline Reversed portal vein blood flow & Gaiani et al.,, ${ }^{37} 1991$ & N/A & 100.0 \\
\hline Increased portal vein congestion index & $\begin{array}{l}\text { Moriyasu et al., }{ }^{29} 1986 \\
\text { Haag et al., }{ }^{36} 1999\end{array}$ & 67.0 & 100.0 \\
\hline $\begin{array}{l}\text { Dilation of the splenic vein and superior mesenteric } \\
\text { vein }\end{array}$ & Goyal et al., ${ }^{38} 1990$ & 72.0 & 100.0 \\
\hline $\begin{array}{l}\text { Reduced respiratory variation of diameter in } \\
\text { splenic and superior mesenteric veins }\end{array}$ & Bolondi et al.., ${ }^{34} 1982$ & 79.7 & 100.0 \\
\hline Splenomegaly & $\begin{array}{l}\text { Berzigotti et al., }{ }^{39} \\
2008\end{array}$ & 93.0 & 36.0 \\
\hline Portosystemic collateral circulation & Vilgrain et al., ${ }^{40} 1990$ & 83.0 & 100.0 \\
\hline Increased Doppler resistive index of splenic artery & $\begin{array}{l}\text { Vizzutti et al., }{ }^{41} 2007 \\
\text { Piscaglia et al., }{ }^{42} 2001\end{array}$ & 84.6 & 70.4 \\
\hline Increased Doppler resistive index of hepatic artery & $\begin{array}{l}\text { Piscaglia et al., }{ }^{42} 2001 \\
\text { Schneider et al., }{ }^{43} \\
1999\end{array}$ & 86.0 & 88.0 \\
\hline Increased Doppler resistive index of renal artery & $\begin{array}{l}\text { Berzigotti et al., }{ }^{39} \\
2008 \\
\text { Vizzutti et al., }{ }^{41} 2007\end{array}$ & 83.6 & 74.4 \\
\hline $\begin{array}{l}\text { Decreased Doppler pulsatility index of superior } \\
\text { mesenteric artery }\end{array}$ & Vizzutti et al., ${ }^{41} 2007$ & 85.7 & 65.2 \\
\hline
\end{tabular}

N/A: not applicable.

With Doppler ultrasound, evaluation of blood haemodynamics can be performed by examining portal vein velocity, congestion index, pulsatility index, and hepatic vein patterns. Measurement of portal vein velocity yields sensitivity of $88 \%$ and specificity of $96 \%$ if the mean portal vein velocity cut-off is $15 \mathrm{~cm} / \mathrm{sec}^{28}$ This approach, however, has high inter-observer and inter-machine variability. In addition, the finding is also influenced by positioning of the patient. Another approach is by measuring congestion index or the ratio of portal vein cross-sectional area and portal velocity. In patients with cirrhosis and portal hypertension, congestion index was 2.5-fold higher compared to healthy patients, with a sensitivity of $67-95 \%{ }^{29}$ In cirrhotic liver, hepatic venous waveform can also be altered due to loss of normal pulsatility in hepatic veins (likely caused by hepatic vein stenosis), which is also correlated with worse survival rate and higher Child-Pugh class. ${ }^{30}$ A study of 121 subjects with NAFLD also demonstrated the potential use of Doppler ultrasound in evaluating hepatic blood flow in NAFLD-related portal hypertension. The authors, however, also addressed the difficulty in reproducing reliable Doppler ultrasound indices as one of the limitations of their study. ${ }^{31}$ Moderate sensitivities have also been shown from increased Doppler resistive index of splenic (84.6\%), hepatic (86\%), and renal (83.6\%) arteries 
obtained from ultrasound-based modalities. The highest specificity, nonetheless, was observed from increased Doppler resistive index of renal artery. ${ }^{5}$

On the other hand, in NAFLD, CEUS has been utilised more often as a modality to distinguish $\mathrm{NASH}$ from steatosis by showing reduced accumulation of contrast microbubbles in NASH. The possibility of using CEUS to detect changes in hepatic vascular parameters has been evaluated by Cocciolillo et al. in a prospective study involving a quantification of portal vein and parenchymal blood flow in NAFLD and NASH subjects. The authors exhibited compelling alteration of vascular flow parameters in subjects with NAFLD and NASH in comparison to the control group by utilising CEUS. All the CEUS procedures were conducted successfully without any adverse events. Nevertheless, further studies in larger population are still necessary to validate this finding. ${ }^{32,33}$

\section{Transient elastography}

Initially, one-dimensional ultrasound TE had only been proposed as a method to assess liver fibrosis. TE utilises the velocity of a low frequency $(50 \mathrm{~Hz})$ elastic shear wave, which is propagated through liver tissue to evaluate liver stiffness. ${ }^{22}$ In a study involving 124 subjects with recurrent hepatitis $C$ virus infection after liver transplantation, the area under the receiver operating characteristic curve (AUROC) of TE for diagnosing CSPH was 0.94 (sensitivity: 90\%; specificity: 81\%; PPV: 81\%; and NPV: 90\%). ${ }^{44}$ Another study by Bureau et al. ${ }^{45}$ demonstrated that performance of TE in diagnosing $\mathrm{CSPH}$ in chronic liver diseases displayed higher cut-off values in alcoholic cirrhosis (34.9 kPa) when compared to viral cirrhosis (20.5 kPa).

The main advantage of using TE is the fact that it is the most widely used and validated modality with a high range of values (2-75 kPa). Despite this, the measurement can be difficult to perform in patients who are obese, patients with narrow intercostal spaces, or patients with massive ascites. TE demonstrated good reproducibility with lack of applicability (80\%), depending on the experience of the operator.,22 In order to overcome these limitations, an effort has been made to combine TE with other noninvasive methods. Berzigotti et al. ${ }^{46}$ evaluated the degree of portal hypertension and the presence of oesophageal varices in 117 subjects using a combination of TE, platelet count, and spleen diameter. The AUROC was higher from the combination (0.909) in comparison to each parameter, suggesting that diagnostic accuracy of TE can be improved when it is combined with other non-invasive modalities. This finding is also supported by a study in NAFLD and NASH subjects, which showed a satisfactory diagnostic performance of the combined use of transient elastography and CEUS. ${ }^{33}$

\section{Shear wave elastography}

The accuracy of shear wave elastography (SWE) is generally comparable with TE in evaluation of liver fibrosis. In a preliminary study performed on subjects with viral hepatitis, it was demonstrated that an acoustic radiation force impulse (ARFI) elastography had similar diagnostic accuracy with TE for diagnosing advanced fibrosis and cirrhosis. ${ }^{47}$ This result is further supported with a meta-analysis showing no significant difference between an ARFI elastography and $T E$ in the detection of liver fibrosis and cirrhosis. ${ }^{48}$ Nevertheless, conflicting results about the superiority of ARFI elastography in diagnosing portal hypertension are still found from previous studies. Attia et al. ${ }^{49}$ discovered that for diagnosing $\mathrm{CSPH}$, the accuracy of liver and spleen stiffness measurement by an ARFI elastography was excellent (area under the curve [AUC] for liver stiffness: 0.929; AUC for spleen stiffness: 0.968) without any significant difference between liver and spleen stiffness measurement $(p=0.79)$. Another study, which compared performance of ARFI and TE in diagnosing $\mathrm{CSPH}$, found a significant correlation between liver stiffness measurement by ARFI $(p<0.001)$ and TE $(p<0.001)$ to HVPG. However, in this study, TE showed higher diagnostic accuracy compared to ARFI (AUC: 0.870 versus 0.855) without any statistically significant difference $(p=0.8) .{ }^{50}$

Similarly, 2D SWE has also been widely used for evaluation of liver fibrosis. A prospective study by Osman et al. ${ }^{51}$ compared the performance of $2 \mathrm{D}$ SWE to TE in 215 subjects with chronic hepatitis, and showed similar accuracy between both modalities. The highest sensitivity and specificity of 2D SWE were observed in evaluation of Stage FO (sensitivity: 91.4\%; specificity: 98.6\%) and 
Stage F4 (sensitivity: 100\%; specificity: 91\%). A review by Jeong et al. ${ }^{52}$ showed a good diagnostic performance of 2D SWE in differentiating between significant fibrosis, advanced fibrosis, and cirrhosis in NAFLD subjects, with an AUROC of $75.0-92.8 \%$. Nevertheless, a relatively high failure rate (2.7-13.0\%) was still observed from the measurement of liver stiffness by this modality, especially in subjects with NAFLD and a higher BMI.

\section{Spleen stiffness measurement}

Evaluation of the association between spleen stiffness measurement (SSM) and HVPG was initially performed in subjects with hepatitis C virus-related cirrhosis by Colecchia et al.,53 in which SSM showed the strongest correlation with HVPG. SSM also demonstrated significantly higher diagnostic accuracy for the presence of oesophageal varices compared to other modalities (AUROC: 0.94). A recent meta-analysis confirmed this finding by showing the superiority of SSM compared to liver stiffness measurement in predicting oesophageal varices (diagnostic odds ratio: 25.73 versus 9.54). SSM also exhibited higher sensitivity (87\%) and specificity (75\%) in adults with chronic liver diseases. ${ }^{54}$

Furthermore, SSM has been proposed as a diagnostic tool for early assessment of portal haemodynamic changes. A study by Santis et al..$^{55}$ observed a significantly reduced SSM $(p<0.001)$ after placement of transjugular intrahepatic portosystemic shunt (TIPS). This study also found that there was no significant correlation between portal atrial gradient and liver stiffness measurement, suggesting the superiority of SSM compared to liver stiffness measurement in monitoring the modification of portal hypertension. On the contrary, Novelli et al. ${ }^{56}$ observed similar number in increased SSM (42\%) and reduced SSM (58\%) after placement of TIPS. Another study of 135 subjects with cirrhosis caused by different aetiologies also demonstrated a stepwise increased diagnostic value of the SSM, in line with increased severity of portal hypertension. Significantly higher average spleen elastography measurement was obtained in subjects with oesophageal varices compared to those without any varices. ${ }^{57} \mathrm{~A}$ review by Colecchia et al. ${ }^{5}$ evaluated the accuracy of spleen stiffness by MRE for detecting CSPH and oesophageal varices, with the best accuracy represented by a cut-off value of $8.8 \mathrm{kPA}$.

\section{Indocyanine green clearance}

Indocyanine green (ICG) is a tricarbocyanine dye with water-soluble characteristics and with an ability to bind to albumin and a-1 lipoproteins. In the past, plasma clearance rate of ICG has a role in pre-operative assessment of the remaining liver tissues. ${ }^{58}$ A study in subjects with compensated liver cirrhosis showed a linear correlation between ICG 15-minute retention test (ICG-r15) with HVP. ICG-r15 also demonstrated a good performance in detection of CSPH (AUC: 0.808). Meanwhile, in diagnosing oesophageal varices, the highest accuracy was shown by ICG-r15 (AUROC: 0.859) compared to other non-invasive parameters. ${ }^{59}$

\section{MRI and CT}

The use of MRI and/or CT is specifically recommended in clinical conditions that require more detailed assessment with accurate visualisation of portal venous system, e.g., portal cholangiopathy in portal cavernoma, evaluation of the extent of thrombosis, ectopic variceal bleeding, and prior to placement of TIPS. In detection of large oesophageal varices, the sensitivity of single-detector and multi-detector CT scan is $84-100 \%$, while the specificity is 90-100\%. Substantial irradiation and moderate inter-observer variability should be considered as the limitations of CT scan., 9,60 Previous evidence also demonstrated a significant correlation between portal pressure and all flow parameters in MRI, with meaningful correlations for portal fraction $(p<0.001)$, portal perfusion $(p<0.001)$, and mean transit time $(p<0.001) .{ }^{61}$

Recent studies have suggested the role of MR-based elastography as an alternative method to evaluate liver elasticity. Data from a singlecentre prospective study of 146 subjects with chronic liver disease showed a significantly higher AUROC value of MRE (0.994) in assessing liver fibrosis compared to ultrasound elastography and aspartate aminotransferase (AST)-to-platelet ratio index (APRI) (AUROC: 0.837 and 0.709, respectively). Interestingly, MRE also exhibited higher diagnostic performance compared to combination of ultrasound elastography and APRI (AUROC: 0.849). ${ }^{62}$ Aside from liver fibrosis, MRE has also emerged as a potential diagnostic modality for assessing portal hypertension. Additional evidence was obtained from another study to assess the performance of 3D multi-frequency MRE for determining the degree 
of portal hypertension and high-risk oesophageal varices. The spleen loss modulus demonstrated the strongest correlation with HVPG compared to other viscoelastic parameters, suggesting it to be the best parameter in the study. ${ }^{54}$

\section{Subharmonic-aided pressure estimation}

Subharmonic-aided pressure estimation (SHAPE) is one of the most recent non-invasive modalities to assess portal pressure. ${ }^{6}$ The basic concept of this technique is using subharmonic emissions from microbubbles to obtain changes in ambient pressure. In general, there are three stages of subharmonic signal generation in incident acoustic power: occurrence, growth, and saturation. SHAPE depends on incident acoustic power in growth stage. An animal study using canines indicated the potential of portal vein pressure monitoring with SHAPE. A statistically significant difference was also observed in subharmonic signal amplitudes prior to and after portal hypertension condition was applied on the canines. ${ }^{63}$

Further evaluation of SHAPE was performed in 45 subjects with chronic liver disease. Twentynine percent of the subjects had NASH as the aetiology of chronic liver disease. There was a strong positive correlation between estimation of SHAPE pressure gradient and HVPG, with the strongest correlation found in the sub-group with HVPG of at least $12 \mathrm{mmHg}$. In addition, significantly higher mean SHAPE gradient was observed in subjects with higher risk of variceal bleeding (AUROC for HVPG $\geq 12 \mathrm{mmHg}$ : 0.94; AUROC for HVPG $\geq 10 \mathrm{~mm}$ : 0.90). The estimated sensitivity and specificity of SHAPE were 100\% and $81 \%$, respectively. Overall, this preliminary study demonstrated the accuracy of SHAPE as a non-invasive tool to measure portal vein pressures in patients with chronic liver disease. ${ }^{65}$

\section{Endoscopic ultrasound-guided measurement of portal pressure gradient}

Previously, an animal study demonstrated comparable results between endoscopic ultrasound (EUS)-guided measurement of PPG and HVPG. Excellent correlation was exhibited between EUS and interventional radiology methods (Pearson's correlation coefficient was 0.999 for all vessels). ${ }^{66}$ Another pilot study in humans with 28 subjects also showed high technical success rate (100\%) in measuring 1.5-19.0 mmHg without any adverse events. ${ }^{67}$ The most recent study by Zhang et al. ${ }^{68}$ also showed similarly high technical success rate $(91.7 \%)$ with similarly good correlation between EUS-guided measurement of PPG and HVPG (Pearson's correlation coefficient was 0.923; $\mathrm{p}<0.001$ ). In this study, no adverse events were reported, suggesting the potential of EUS-guided measurement of PPG as a direct, safe, and accurate method in assessing portal hypertension.

\section{COMBINATION OF NON-INVASIVE BIOMARKERS FOR NON-ALCOHOLIC FATTY LIVER DISEASE-RELATED PORTAL HYPERTENSION}

The role of non-invasive biomarkers has also been emphasised in NAFLD and NASH to substitute liver biopsy as the main standard reference for assessment of the severity of NAFLD. ${ }^{69}$ Considering the invasive nature of liver biopsy, as well as the risk of sampling bias, it is necessary to find other methods to overcome those limitations. Several indices and biomarker panels have been proposed as alternative options (Table 3). Moderate accuracy has been demonstrated by the Fatty Liver Index (FLI), which consists of BMI, waist circumference, and serum triglyceride and $\mathrm{V}$-glutamyl transferase levels. The FLI has shown sensitivity as high as $87 \%$ and AUROC of 0.84 in diagnosing fatty liver, although this test cannot be used to differentiate steatosis grades.69 FLI, however, has been demonstrated to have independent association with liver-related mortality within 15 years (hazard ratio: 1.04) in an Italian cohort study. Moreover, FLI was also significantly associated with fibrinogen level, which was considered as a surrogate marker of inflammation. ${ }^{70}$ Another option that can be considered is the Hepatic Steatosis Index (HSI), which consists of the ratio between serum AST and serum alanine aminotransferase (ALT), sex, BMI, and history of diabetes mellitus. Moderate accuracy (AUROC: 0.801-0.824) has been shown by HSI with high sensitivity (93.1\%) and specificity (92.4\%). ${ }^{69,71}$ Another retrospective observational study in a population with HIV infection also validated the performance of $\mathrm{HSI}$ in diagnosing steatosis (diagnostic accuracy: $84.5 \%)^{72}$ 
Table 3: Summary of potential non-invasive biomarker panels for non-alcoholic fatty liver disease-related portal hypertension.

\begin{tabular}{|c|c|c|c|c|c|c|c|}
\hline $\begin{array}{l}\text { Non- } \\
\text { invasive } \\
\text { panels }\end{array}$ & References & $\begin{array}{l}\text { Sensitivity } \\
(\%)\end{array}$ & $\begin{array}{l}\text { Specificity } \\
(\%)\end{array}$ & PPV & NPV & AUROC & Limitations \\
\hline FLI & $\begin{array}{l}\text { Wong et } \\
\text { al., } 692018 \\
\text { Calori et } \\
\text { al., }^{70} 2011\end{array}$ & 87.0 & 64.0 & $\mathrm{~N} / \mathrm{A}$ & $N / A$ & $\begin{array}{l}\text { O.84 (for fatty } \\
\text { liver diagnosis) } \\
\text { Statistically } \\
\text { significant } \\
\text { independent } \\
\text { association with } \\
\text { liver inflammation } \\
\text { and liver-related } \\
\text { deaths within } 15 \\
\text { years }\end{array}$ & $\begin{array}{l}\text { Sub-optimal } \\
\text { reference } \\
\text { standards } \\
\text { (ultrasonography } \\
\text { findings may } \\
\text { be operator- } \\
\text { dependent and } \\
\text { show lower } \\
\text { sensitivity towards } \\
\text { lower grade of } \\
\text { steatosis) }\end{array}$ \\
\hline $\mathrm{HSI}$ & $\begin{array}{l}\text { Wong et } \\
\text { al., }^{69} 2018 \\
\text { Lee et al., } \\
2010 \\
\text { Sebastiani } \\
\text { et al., } \\
2015\end{array}$ & 93.1 & 92.4 & $\mathrm{~N} / \mathrm{A}$ & N/A & $\begin{array}{l}\text { O.801-0.824 (for } \\
\text { NAFLD screening) } \\
\text { O.845 (for } \\
\text { predicting hepatic } \\
\text { steatosis in HIV } \\
\text { mono-infection) }\end{array}$ & $\begin{array}{l}\text { Sub-optimal } \\
\text { reference } \\
\text { standards } \\
\text { (ultrasonography } \\
\text { findings may } \\
\text { be operator- } \\
\text { dependent and } \\
\text { show lower } \\
\text { sensitivity towards } \\
\text { lower grade of } \\
\text { steatosis) }\end{array}$ \\
\hline NFS & $\begin{array}{l}\text { Lee et al., } \\
2010\end{array}$ & $N / A$ & N/A & 44 & 93 & $\begin{array}{l}0.70-0.83 \text { (for } \\
\text { predicting liver- } \\
\text { related events) } \\
0.65-0.83 \text { (for } \\
\text { predicting fibrosis } \\
\text { progression) }\end{array}$ & $\begin{array}{l}\text { Varied BMI } \\
\text { interpretation } \\
\text { among different } \\
\text { ethnicities }\end{array}$ \\
\hline FIB-4 & $\begin{array}{l}\text { Lee et al., } \\
2010\end{array}$ & $N / A$ & $N / A$ & 49 & 97 & $\begin{array}{l}0.67-0.82 \text { (for } \\
\text { predicting liver- } \\
\text { related events) } \\
0.65-0.81 \text { (for } \\
\text { predicting fibrosis } \\
\text { progression) }\end{array}$ & $\begin{array}{l}\text { Moderate } \\
\text { reproducibility } \\
\text { because } \\
\text { aminotransferases } \\
\text { can fluctuate } \\
\text { rapidly }\end{array}$ \\
\hline APRI & $\begin{array}{l}\text { Lee et al.,71 } \\
2010 \\
\text { Siddiqui et } \\
\text { al., }^{73} 2019\end{array}$ & $N / A$ & N/A & 47 & 97 & $\begin{array}{l}0.52-0.73 \text { (for } \\
\text { predicting liver- } \\
\text { related events) } \\
0.65-0.82 \text { (for } \\
\text { predicting fibrosis } \\
\text { progression) }\end{array}$ & Modest accuracy \\
\hline BARD & $\begin{array}{l}\text { Harrison et } \\
\text { al., }{ }^{74} 2008\end{array}$ & $N / A$ & $N / A$ & 42 & 97 & $\begin{array}{l}\text { O.81 (for } \\
\text { predicting fibrosis } \\
\text { Stage } 3-4 \text { ) }\end{array}$ & $\begin{array}{l}\text { Varied BMI } \\
\text { interpretation } \\
\text { among different } \\
\text { ethnicities }\end{array}$ \\
\hline
\end{tabular}




\begin{tabular}{|c|c|c|c|c|c|c|c|}
\hline $\begin{array}{l}\text { Non- } \\
\text { invasive } \\
\text { panels }\end{array}$ & References & $\begin{array}{l}\text { Sensitivity } \\
(\%)\end{array}$ & $\begin{array}{l}\text { Specificity } \\
(\%)\end{array}$ & PPV & NPV & AUROC & Limitations \\
\hline $\begin{array}{l}\text { ARFI- } \\
\text { spleen } \\
\text { diameter- } \\
\text { to-platelet } \\
\text { ratio }\end{array}$ & $\begin{array}{l}\text { Park et al., }{ }^{75} \\
2015\end{array}$ & $\begin{array}{l}81.1 \text { (for } \\
\text { diagnosis of } \\
\text { oesophageal } \\
\text { varices) } \\
90.0 \text { (for } \\
\text { high-risk } \\
\text { varices) }\end{array}$ & $\begin{array}{l}84.0 \text { (for } \\
\text { diagnosis of } \\
\text { oesophageal } \\
\text { varices) } \\
94.3 \text { (for } \\
\text { high-risk } \\
\text { varices) }\end{array}$ & $\begin{array}{l}63.8 \text { (for } \\
\text { diagnosis of } \\
\text { oesophageal } \\
\text { varices) } \\
72.0 \text { (for high- } \\
\text { risk varices) }\end{array}$ & $\begin{array}{l}92.7 \text { (for } \\
\text { diagnosis of } \\
\text { oesophageal } \\
\text { varices) } \\
98.3 \text { (for high- } \\
\text { risk varices) }\end{array}$ & $\begin{array}{l}0.903 \text { (for } \\
\text { diagnosis of } \\
\text { oesophageal } \\
\text { varices) } \\
\text { 0.946 (for high- } \\
\text { risk varices }\end{array}$ & $\begin{array}{l}\text { Relatively lower } \\
\text { PPV indicates } \\
\text { that unnecessary } \\
\text { endoscopic } \\
\text { procedures may } \\
\text { be performed in } \\
\text { some patients } \\
\text { without high-risk } \\
\text { varices }\end{array}$ \\
\hline Hepascore & $\begin{array}{l}\text { Huang et } \\
\text { al.., }^{76} 2017\end{array}$ & N/A & $\mathrm{N} / \mathrm{A}$ & $\mathrm{N} / \mathrm{A}$ & $\mathrm{N} / \mathrm{A}$ & $\begin{array}{l}0.92 \text { (for } \\
\text { predicting } \\
\text { cirrhosis) } \\
0.83 \text { (for } \\
\text { predicting } \\
\text { advanced fibrosis) }\end{array}$ & $\begin{array}{l}\text { Heterogeneity } \\
\text { between studies }\end{array}$ \\
\hline ELF Test & $\begin{array}{l}\text { Vali et al..,7 } \\
2020\end{array}$ & $\begin{array}{l}93 \text { (for cut- } \\
\text { off score: } \\
7.70 \text { ) } \\
36-65 \text { (for } \\
\text { cut-off } \\
\text { scores: 9.80, } \\
10.51,11.30 \text { ) }\end{array}$ & $\begin{array}{l}34 \text { (for cut- } \\
\text { off score: } \\
7.70 \text { ) } \\
86-96 \text { (for } \\
\text { cut-off } \\
\text { scores: } 9.80 \text {, } \\
\text { 10.51, 11.30) }\end{array}$ & $\mathrm{N} / \mathrm{A}$ & $\begin{array}{l}0.83-0.98 \\
\text { (in settings } \\
\text { with disease } \\
\text { prevalence } \\
<40 \% \text { ) }\end{array}$ & 0.81 & $\begin{array}{l}\text { Limited sensitivity } \\
\text { for excluding } \\
\text { advanced and } \\
\text { significant fibrosis } \\
\text { with higher cut-off } \\
\text { scores }\end{array}$ \\
\hline FAST & $\begin{array}{l}\text { Newsome } \\
\text { et al.,78 } \\
2020\end{array}$ & $\begin{array}{l}64-100 \text { (for } \\
\text { rule-out } \\
\text { zone) } \\
4-36 \text { (for } \\
\text { rule-in zone) }\end{array}$ & $\begin{array}{l}\text { 35-86 (for } \\
\text { rule-out } \\
\text { zone) } \\
25-75 \text { (for } \\
\text { rule-in zone) }\end{array}$ & $\begin{array}{l}\text { 33-83 (for } \\
\text { rule-in zone) }\end{array}$ & $\begin{array}{l}73-100 \text { (for } \\
\text { rule-out zone) }\end{array}$ & $0.74-0.95$ & $\begin{array}{l}\text { Higher expertise } \\
\text { and facilities may } \\
\text { be needed to } \\
\text { utilise this method } \\
\text { in primary care }\end{array}$ \\
\hline
\end{tabular}

APRI: AST to Platelet Ratio Index; AST: aspartate aminotransferase; AUROC: area under the receiver operating characteristic curve; ELF: enhanced liver fibrosis; FAST: FibroScan-AST; FIB-4: Fibrosis-4; FLI: Fatty Liver index; HIS: Hepatic Steatosis Index; N/A: not applicable; NAFLD: non-alcoholic fatty liver disease; NFS: NAFLD Fibrosis Score; NPV: negative predictive value; PPV: positive predictive value.

To date, the most common non-invasive panels to be utilised are the NAFLD Fibrosis Score (NFS), Fibrosis-4 (FIB-4), APRI, and BARD score. NFS consists of age, BMI, diabetes mellitus, ratio of serum AST and serum ALT, platelet count, and albumin. This parameter has been validated with AUROC of $0.82 .^{79}$ On the other hand, FIB-4 index (which includes age, AST, platelet count, and ALT components) and APRI score (which includes AST and platelet count components), which were first introduced in 2003 and 2006, respectively, have also shown promising results in previous studies. ${ }^{80} \mathrm{~A}$ systematic review by Lee et al. ${ }^{23}$ pointed out that FIB-4, NFS, and APRI showed good prognostic accuracy for liver-related events with AUROC ranging from 0.69 to 0.92. However, FIB-4 and NFS showed superior results (AUROC: 0.67-0.82 and 0.7-0.83, respectively) compared to APRI (AUROC: 0.52-0.73) in predicting mortality. Higher accuracy for predicting fibrosis progression was also shown by FIB-4 (AUROC: 0.65-0.81) and NFS (AUROC: 0.65-0.83) in comparison to APRI (AUROC: 0.65-0.82). A 
cross-sectional study by Siddiqui et al. ${ }^{73}$ showed low PPVs and high NPVs in FIB-4, NFS, and APRI. Meanwhile, BARD score consists of $\mathrm{BMI}$, ratio of serum AST and ALT, and diabetes mellitus. The AUROC was 0.81 for detection of Stage 3-4 fibrosis, with PPV and NPV of $42 \%$ and $97 \%$, respectively. ${ }^{74}$

Several other models are also currently emerging as potential diagnostic tools. A combination of platelet count and ARFI elastography was also used to produce a novel model, called ARFI-spleen diameter-to-platelet-ratio score, for evaluating oesophageal varices. The use of ARFI-spleen diameter-to-platelet-ratio score demonstrated superiority compared to ARFI elastography alone in diagnosis of oesophageal varices (AUROC: 0.769) and in high-risk varices (diagnostic accuracy: 93.5\%). ${ }^{77}$ A serum model called Hepascore, which was initially developed to predict the severity of liver fibrosis in chronic hepatitis C, consists of a2-macroglobulin, hyaluronic acid, and $\mathrm{V}$-glutamyl transpeptidase. One meta-analysis showed excellent performance of Hepascore in predicting cirrhosis (AUROC: 0.92) and advanced fibrosis (adjusted AUROC: 0.83). ${ }^{76}$ Another proposed non-invasive method is enhanced liver fibrosis (ELF) test (consists of Type III procollagen peptide, hyaluronic acid, and tissue inhibitor of metalloproteinase-1), which exhibited moderate accuracy (AUROC: 0.81) and high sensitivity (97\%) in differentiating significant fibrosis in subjects with NAFLD located in high prevalence settings. ${ }^{77}$ A new diagnostic score under development, called FibroScan ${ }^{\circledR}$-AST $\left(\right.$ FAST $^{\mathrm{TM}}$ ) score (Echosens, Paris, France), which combines liver stiffness measurement, controlled attenuation parameter, and AST, also indicated a satisfactory performance in subjects with NASH (AUROC: 0.80). ${ }^{78}$

\section{POTENTIAL FUTURE THERAPEUTIC OPTIONS FOR NON-ALCOHOLIC FATTY LIVER DISEASE-RELATED PORTAL HYPERTENSION}

Aside from lifestyle intervention, various therapeutic targets from the cellular and molecular pathophysiology of NAFLD have been addressed from previous clinical evidence, especially with the therapeutic goal of decreasing intrahepatic vascular resistance. One of the greatest-potential therapeutic targets is sinusoid vascular regulation. Statins, in particular, have been proposed as the safest and the most effective agent to repair sinusoidal microvascular dysfunction. The involvement of statins is highlighted in eNOS-NO-sGC-cGMP, signalling through the up-regulation of transcription factor KLF2 and inhibition of Ras homolog family member A/Rho-associated coiled-coil protein kinase (RhoA/ROCK) pathway. The RhoA/ROCK pathway can cause vasoconstriction through several processes, such as regulating cytoskeletal structures for liver sinusoid endothelial cells capillarisation and increasing phosphorylation of myosin light chains. Another potential agent with similar therapeutic goal is nuclear farnesoid $X$ receptor agonists. This agent is able to lower intrahepatic vasoconstriction by stimulating endothelial nitric oxide synthase activity, inhibiting contraction of stellate cells, inducing degradation of asymmetric dimethylarginine, and up-regulating the expression of cystathionase. Moreover, nuclear farnesoid $X$ receptor agonist has also been shown to reduce de novo lipogenesis. ${ }^{10}$

Several other sinusoidal pathophenotypes have also been addressed as potential therapeutic targets in NAFLD-related portal hypertension. As a multi-kinase inhibitor, sorafenib has been demonstrated to be capable of reducing portal hypertension in cirrhosis by inhibiting the activation of vascular endothelial growth factor and platelet-derived growth factor. The use of anticoagulant has also been considered in tackling the microthrombosis problem, which may be involved in the development of portal hypertension. The use of antibiotics (e.g., rifaximin), antioxidant, and anti-inflammatory drugs have also been utilised to manage the dynamic components of vascular resistance in NAFLD. ${ }^{10}$

\section{CONCLUSION}

As a growing and significant global health problem, progression and complications of NAFLD remain the most essential fields to be studied, particularly because development of portal hypertension in NAFLD can occur without any extensive fibrosis or cirrhosis. Currently, HVPG is still the gold standard for measuring portal pressure, including in patients with NAFLD-related hypertension. However, recent 
evidence demonstrated that the opportunity to optimise the gold standard techniques with other non-invasive assessments should not be overlooked, especially in non-cirrhotic portal hypertension cases. Further studies are crucially required to compare the accuracy and costeffectiveness of potential non-invasive tests for
NAFLD-related portal hypertension. Currently, some potential non-invasive modalities, i.e., combined novel biomarkers and/or metabolomic, are still under development. EUS-guided measurement of PPG is also considered as a direct, safe, and accurate method in assessing NAFLD-related portal hypertension.

\section{References}

1. Younossi ZM et al. Epidemiology of nonalcoholic fatty liver disease and nonalco-holic steatohepatitis: implications for liver transplantation. Transplantation. 2019;103(1):22-7.

2. Mitra $\mathrm{S}$ et al. Epidemiology of non-alcoholic and alcoholic fatty liver diseases. Transl Gastroentero Hepatol. 2020;5:16.

3. Buzzetti $E$ et al. The multiple-hit pathogenesis of non-alcoholic fatty liver disease (NAFLD). Metabolism. 2016;65(8):1038-48.

4. Groszmann RJ, Wongcharatrawee S The hepatic venous pressure gradient: anything worth doing should be done right. Hepatology. 2004;39(2):280-3.

5. Colecchia A et al. Liver and spleen stiffness and other methods to assess portal hypertension in cirrhotic patients: a review of the literature. Eur J Gastroenterol Hepatol. 2015;27(9):992-1001.

6. Bolognesi $M$ et al. Clinical role of non-invasive assessment of portal hypertension. World J Gastroenterol. 2017;23(1):1-10.

7. Castera L et al. Non-invasive evaluation of portal hypertension using transient elastography. J Hepatol. 2012;56(3):696-703.

8. Stefanescu H, Procopet B. Noninvasive assessment of portal hypertension in cirrhosis: liver stiffness and beyond. World J Gastroenterol. 2014;20(45):16811-9.

9. Berzigotti A et al. Assessing portal hypertension in liver diseases. Expert Rev Gastroenterol Hepatol. 2013;7(2):141-55.

10. Ryou M et al. Nonalcoholic fatty liver disease and portal hypertension. Explor Med. 2020;1:149-69.

11. Moga $L$ et al. Patients with NAFLD do not have severe portal hypertension in the absence of cirrhosis. J Hepatol. 2021;74(5):1269-70.

12. Mendes FD et al. Prevalence and indicators of portal hypertension in patients with nonalcoholic fatty liver disease. Clin Gastroenterol Hepatol. 2012;10(9):1028-33.e2.

13. Semmler $\mathrm{G}$ et al. The impact of hepatic steatosis on porta hypertension. PLoS One. 2019;14(11):e0224506.

14. Da BL et al. Portal pressure in noncirrhotic portal hypertension: to measure or not to measure. Hepatology. 2019;70(6):2228-30.

15. Navin PJ et al. The role of magnetic resonance elastography in the diagnosis of noncirrhotic portal hypertension. Clin Gastroenterol Hepatol. 2020;18(13):3051-3.e2.

16. Gharib AM et al. Magnetic resonance elastography shear wave velocity correlated with liver fibrosis and hepatic venous pressure gradient in adults with advanced liver disease. Biomed Res Int. 2017;2017;2067479.

17. Cheng $\mathrm{J}$ et al. Metabolomics: a highthroughput platform for metabolite profile exploration. Methods Mol Biol. 2018;1754:265-91.

18. Grønbaek H et al. Soluble CD163, a marker of Kupffer cell activation, is related to portal hypertension in patients with liver cirrhosis. Aliment Pharmacol Ther. 2012;36(2):173-80.

19. Qi $X$ et al. Emerging non-invasive approaches for diagnosis and monitoring of portal hypertension. Lancet Gastroenterol Hepatol. 2018;3(10):708-19.

20. Ravaioli $F$ et al. Non-invasive assessment of portal hypertension in chronic liver disease: an update. Gastroenterol Res Pract. 2018;2018:4202091.

21. Sabri SS, Saad WEA. Anatomy and classification of gastrorenal and gastrocaval shunts. Semin Intervent Radiol. 2011;28(3):296-302.

22. European Association for the Study of the Liver, Asociacion Latinoamericana para el Estudio del Higado. EASL-ALEH clinical practice guidelines - non-invasive tests for evaluation of liver disease severity and prognosis. J Hepatol. 2015;63(1):237-64

23. Lee $\mathrm{J}$ et al. Prognostic accuracy of FIB-4, NAFLD fibrosis score, and APRI for NAFLD-related events: a systematic review. Liver Int. 2021;41(2):261-70.

24. Kamath PS, Mookerjee RP. Individualized care for portal hypertension: not quite yet. Hepatol. 2015;63(3):543-5.
25. Laurain A et al. Oesophageal capsule endoscopy versus oesophagogastroduodenoscopy for the diagnosis of recurrent varices: a prospective multicenter study. Dig Liver Dis. 2014;46(6):535-40.

26. Sacher-Huvelin S et al. Screening of esophageal varices by esophageal capsule endoscopy: results of a French multicenter prospective study. Endoscopy. 2015;47(6):486-92

27. Robinson KA et al. Doppler sonography of portal hypertension. Ultrasound Q. 2009;25(1):3-13.

28. Zironi G et al. Value of measurement of mean portal flow velocity by Doppler flowmetry in the diagnosis of portal hypertension. J Hepatol. 1992;16(3):298-303.

29. Moriyasu F et al. "Congestion index" of the portal vein. AJR Am J Roentgenol. 1986;146(4):735-9.

30. Kawanaka $\mathrm{H}$ et al. Abnormality of the hepatic vein waveforms in cirrhotic patients with portal hypertension and its prognostic implications. J Gastroenterol Hepatol. 2007;23(7 Pt 2):e129-36.

31. Hirooka $\mathrm{M}$ et al. Nonalcoholic fatty liver disease: portal hypertension due to outflow block in patients without cirrhosis. Radiology. 2015;274(2):597604.

32. Ballestri $\mathrm{S}$ et al. Role of ultrasound in the diagnosis and treatment of nonalcoholic fatty liver disease and its complications. Expert Rev Gastroenterol Hepatol. 2015;9(5):60327.

33. Cocciolillo $\mathrm{S}$ et al. CEUS and Fibroscan in non-alcoholic fatty liver disease and non-alcoholic steatohepatitis. World J Hepatol. 2014;6(7):496-503.

34. Bolondi $L$ et al. Ultrasonography in the diagnosis of portal hypertension: diminished response of portal vessels to respiration. Radiology. 1982;142(1):167-72.

35. Zironi G et al. Value of measurement of mean portal flow velocity by Doppler flowmetry in the diagnosis of portal hypertension. J Hepatol. 1992;16(3):298-303.

36. Haag $\mathrm{K}$ et al. Correlation of duplex sonography findings and portal 
pressure in 375 patients with portal hypertension. AJR Am J Roentgenol. 1999;172(3):631-5.

37. Gaiani S et al. Prevalence of spontaneous hepatofugal portal flow in liver cirrhosis: clinical and endoscopic correlation in 228 patients. Gastroenterology. 1991;100(1):160-7.

38. Goyal AK et al. Ultrasonic measurements of portal vasculature in diagnosis of portal vasculature in diagnosis of portal hypertension. A controversial subject reviewed. J Ultrasound Med. 1990;9(1):45-8.

39. Berzigotti A et al. Spleen enlargement on follow-up evaluation: a noninvasive predictor of complications of portal hypertension in cirrhosis. Clin Gastroenterol Hepatol. 2008;6(10):1129-34.

40. Vilgrain $\vee$ et al. Comparison between ultrasonographic signs and the degree of portal hypertension in patients with cirrhosis. Gastrointest Radiol. 1990;15:218-22.

41. Vizzutti F et al. Performance of Doppler ultrasound in the prediction of severe portal hypertension in hepatitis $C$ virus-related chronic liver disease. Liver Int. 2007;27(10):137988.

42. Piscaglia F et al. Value of splanchnic Doppler ultrasound in the diagnosis of portal hypertension. Ultrasound Med Biol. 2001;27(7):893-9.

43. Schneider AW et al. Hepatic arterial pulsatility index in cirrhosis: correlation with portal pressure. J Hepatol. 1999;30(5):876-81.

44. Carrión JA et al. Transient elastography for diagnosis of advanced fibrosis and portal hypertension in patients with hepatitis $C$ recurrence after liver transplantation. Liver Transpl. 2006;12(12):1791-8.

45. Bureau $\mathrm{C}$ et al. Transient elastography accurately predicts presence of significant portal hypertension in patients with chronic liver disease. Aliment Pharmacol Ther. 2008;27(12):1261-8.

46. Berzigotti A et al. Elastography, spleen size, and platelet count identify portal hypertension in patients with compensated cirrhosis. Gastroenterology. 2013;144(1):102-11. e1.

47. Friedrich-Rust $M$ et al. Liver fibrosis in viral hepatitis: non-invasive assessment with acoustic radiation force impulse imaging versus transient elastography. Radiology. 2009;252(2):595-604

48. Bota $S$ et al. Meta-analysis: ARFI elastography versus transient elastography for the evaluation of liver fibrosis. Liver Int. 2013;33(8):1138-47.

49. Attia $D$ et al. Evaluation of liver and spleen stiffness with acoustic radiation force impulse quantification elastography for diagnosing clinically significant portal hypertension. Ultraschall Med. 2015;36(6):603-10.

50. Salzi P et al. Evaluation of portal hypertension and varices by acoustic radiation force impulse imaging of the liver compared to transient elastography and AST to platelet ratio index. Ultraschall Med. 2014;35(6):528-33.

51. Osman AM et al. 2D shear wave elastography (SWE) performance versus vibration-controlled transient elastography (VCTE/fibroscan) in the assessment of liver stiffness in chronic hepatitis. Insights Imaging 2020;11(1):38

52. Jeong JY et al. Role of twodimensional shear wave elastography in chronic liver diseases: a narrative review. World J Gastroenterol. 2018;24(34):3849-60.

53. Colecchia A et al. Measurement of spleen stiffness to evaluate portal hypertension and the esophageal varices in patients with HCVrelated cirrhosis. Gastroenterology. 2012;143(3):646-54

54. Ma X et al. Spleen stiffness is superior to liver stiffness for predicting esophageal varices in chronic liver disease: a meta-analysis. PLoS One. 2016;11(11):e0165786.

55. Santis A et al. Modification of splenic stiffness on acoustic radiation force impulse parallels the variation of portal pressure induced by transjugular intrahepatic portosystemic shunt. J Gastroenterol Hepatol. 2017;33(3):704-9.

56. Novelli PM et al. Sonographic assessment of spleen stiffness before and after transjugular intrahepatic portosystemic shunt placement with or without concurrent embolization of portal systemic collateral veins in patients with cirrhosis and portal hypertension: feasibility study. J Ultrasound Med. 2015;34(3):443-9.

57. Fierbinteanu-Braticevici $\mathrm{C}$ et al. Spleen stiffness as predictor of esophageal varices in cirrhosis of different etiologies. Sci Rep. 2019;9(1):16190.

58. Yokoyama $Y$ et al. Value of indocyanine green clearance of the future liver remnant in predicting outcome after resection for biliary cancer. Br J Surg. 2010;97(8):1260-8.

59. Lisotti $A$ et al. Indocyanine green retention test as a non-invasive marker of portal hypertension and esophageal varices in compensated liver cirrhosis. Hepatology. 2014;59(2):643-50.

60. Kim H et al. Evaluation of esophagea varices on liver computed tomography: receiver operating characteristic analyses of the performance of radiologists and endoscopists. J Gastroenterol Hepatol. 2009;24(2009):1534-40.

61. Annet $L$ et al. Hepatic flow parameters measured with MR imaging and Doppler US: correlations with degree of cirrhosis and portal hypertension. Radiology. 2003;229(2):409-14

62. Huwart L et al. Magnetic resonance elastography for the noninvasive staging of liver fibrosis. Gastroenterology. 2008;135(1):32-40.

63. Ronot $\mathrm{M}$ et al. Assessment of portal hypertension and high-risk oesophageal varices with liver and spleen three-dimensional multifrequency MR elastography in liver cirrhosis. Eur Radiol. 2014;24(6):1394-402.

64. Dave JK et al. Investigating the efficacy of subharmonic aided pressure estimation for portal vein pressures and portal hypertension monitoring. Ultrasound Med Biol. 2012;38(10):1784-98

65. Eisenbrey JR et al. Chronic liver disease: noninvasive subharmonic aided pressure estimation of hepatic venous pressure gradient. Radiology. 2013:268(2):581-8.

66. Huang JY et al. EUS-guided portal pressure gradient measurement with a novel 25 -gauge needle device versus standard transjugular approach: a comparison animal study. Gastrointest Endosc. 2016;84(2):35862.

67. Huang JY et al. EUS-guided portal pressure gradient measurement with a simple novel device: a human pilot study. Gastrointest Endosc. 2017;85(5):996-1001.

68. Zhang $W$ et al. EUS-guided portal pressure gradient measurement in patients with acute or subacute portal hypertension. Gastrointest Endosc. 2021;93(3):565-72.

69. Wong $V W S$ et al. Non-invasive biomarkers in NAFLD and NASH current progress and future promises. Nat Rev Gastroenterol Hepatol. 2018;15(8):461-78.

70. Calori $\mathrm{G}$ et al. Fatty liver index and mortality: the cremona study in the 15th year of follow-up. Hepatology. 2011;54(1):145-52.

71. Lee $\mathrm{JH}$ et al. Hepatic steatosis index a simple screening tool reflecting nonalcoholic fatty liver disease. Dig Liver Dis. 2010;42(7):503-8.

72. Sebastiani $\mathrm{G}$ et al. Incidence and predictors of hepatic steatosis and fibrosis by serum biomarkers in a large cohort of human immunodeficiency virus monoinfected patients. Open Forum Infect Dis. 2015;2(1):ofv015.

73. Siddiqui MS et al. Diagnostic accuracy of noninvasive fibrosis models to detect change in fibrosis stage. Clin Gastroenterol Hepatol. 
2019;17(9):1877-85.e5.

74. Harrison SA et al. Development and validation of a simple NAFLD clinical scoring system for identifying patients without advanced disease. Gut. 2008;57(10):1441-7.

75. Park $Y$ et al. A novel model to predict esophageal varices in patients with compensated cirrhosis using acoustic radiation force impulse elastography. PLoS One. 2015;10(3):e0121009.

76. Huang $Y$ et al. The ability of Hepascore to predict liver fibrosis in chronic liver disease: a meta-analysis. Liver Int. 2017;37(1):121-31.

77. Vali $Y$ et al. Enhanced liver fibrosis test for the non-invasive diagnosis of fibrosis in patients with NAFLD: a systematic review and meta-analysis. J Hepatol. 2020;73(2):252-62.

78. Newsome PN et al. FibroScan-AST (FAST) score for the non-invasive identification of patients with non-alcoholic steatohepatitis with significant activity and fibrosis: a prospective derivation and globa validation study. Lancet Gastroenterol Hepatol. 2020;5(4):362-73.

79. Angulo $P$ et al. The NAFLD fibrosis score: a noninvasive system that identifies liver fibrosis in patients with NAFLD. Hepatology. 2007;45(4):84654

80. Seipalla GE et al. Analysis of liver fibrosis degree with APRI score and FIB-4 index patients with nonalcoholic fatty liver disease. Indones J Clinical Pathol Med Laboratory 2020;26(2):158-61. 